\title{
X-ray precursors to flares and filament eruptions ${ }^{\star}$
}

\author{
C. Chifor ${ }^{1}$, D. Tripathi ${ }^{1}$, H. E. Mason ${ }^{1}$, and B. R. Dennis ${ }^{2}$ \\ 1 Department of Applied Mathematics and Theoretical Physics, Centre for Mathematical Sciences, Wilberforce Road, \\ Cambridge CB3 0WA, UK \\ e-mail: [c.chifor;d.tripathi;h.e.mason] @damtp.cam.ac.uk \\ 2 NASA Goddard Space Flight Center, Greenbelt, MD 20771, USA \\ e-mail: brian.r.dennis@nasa.gov
}

Received 2 May 2007 / Accepted 22 June 2007

\begin{abstract}
Aims. To study preflare X-ray brightenings as diagnostics of the destabilisation of flare-associated erupting filaments/prominences. Methods. We combine new observations from the Transition Region and Coronal Explorer (TRACE) and the Reuven Ramaty High Energy Solar Spectroscopic Imager (RHESSI), as well as revisit events reported in the literature to date, in order to scrutinise the preflare activity during eight flare-associated filament eruptions.

Results. The preflare activity occurs in the form of discrete, localised X-ray brightenings observed between 2 and 50 min before the impulsive phase of the flare and filament acceleration. These transient preflare brightenings are situated on or near (within 10" of) the polarity inversion line (PIL), coincident with emerging and/or canceling magnetic flux. The filaments begin to rise from the location of the preflare brightenings. In five out of eight events, the preflare brightenings were observed beneath the filament channel, close to the filament footpoint first observed to rise. Both thermal and nonthermal hard X-ray emissions during the preflare enhancement were detected with RHESSI, suggesting that both plasma heating and electron acceleration occurred at this time. The main energy release during the impulsive phase of the flare is observed close to (within $50^{\prime \prime}$ of) the preflare brightenings. The fast-rise phase of the filament eruption starts at the same time as the onset of the main flare or up to 5 min later.

Conclusions. The preflare brightenings are precursors to the flare and filament eruption. These precursors represent distinct, localised instances of energy release, rather than a gradual energy release prior to the main flare. The X-ray precursors represent clearly observable signatures in the early stages of the eruption. Together with the timing of the filament fast-rise at or after the main flare onset, the X-ray precursors provide evidence for a tether-cutting mechanism initially manifested as localised magnetic reconnection being a common trigger for both flare emission and filament eruption.
\end{abstract}

Key words. Sun: filaments - Sun: prominences - Sun: coronal mass ejections (CMEs) - Sun: flares - Sun: UV radiation Sun: X-rays, gamma rays

\section{Introduction}

Preflare activity such as transient brightenings before the main impulsive phase of a flare has long been considered a potential clue to an understanding of the physical conditions in the solar atmosphere that lead to an eruption. Bumba \& Křiviský (1959), who introduced the term "preflares", were the first to address this topic. Reviews of early studies for such flare precursors can be found in Martin et al. (1980) and Van Hoven \& Hurford (1984).

Transient brightenings at the onset of solar flares have been identified across the electromagnetic spectrum: in radio (Kai et al. 1983; Fárník et al. 2002), H $\alpha$ (e.g. Contarino et al. 2003), UV and EUV (e.g. OV emission - Cheng et al. 1985; $1600 \AA$ continuum emission - Warren \& Warshall 2001). An early comprehensive review of the multiwavelength preflare activity was given by Schmahl et al. (1989). From X-ray observations taken with the Hard X-ray imaging spectrometer (HXIS) on board the Solar Maximum Mission (SMM), Harrison et al. (1985) and Tappin (1991) found soft X-ray (SXR) emission preceding the majority of flares, but spatial information to relate this preflare activity

$\star$ Movies and Appendix A are only available in electronic form at http://www. aanda.org to the site of the main energy release was missing for most analysed events. Using Yohkoh/Soft X-ray Telescope (SXT) images, Fárník et al. (1998) addressed the spatial relations between the preflare activity and flares, finding the flaring loops brightening tens of minutes before the impulsive flare phase in $25 \%$ of their events. Several authors have shown evidence of hard X-ray (HXR) nonthermal emission prior to the flare impulsive phase (e.g. Fárník et al. 2002; Holman et al. 2003; Asai et al. 2006; Chifor et al. 2006; Sui et al. 2006) and spectral line broadening (e.g. Cheng 1990).

It is widely accepted that solar eruptive events are of magnetic origin and have three manifestations: flare, filament eruption, and coronal mass ejection (CME), with all three in close association (e.g. Shibata 1999; Priest \& Forbes 2002; Svestka 2001). The pre-eruption conditions are important not only for the initiation of flares, but also for all accompanying eruptive phenomena. Understanding the pre-eruption conditions could shed new light on one of the most important puzzle of space weather which is understanding why flares, filaments, and CMEs erupt.

Early evidence of discrete SXR precursors approximately 15-30 min before the linearly extrapolated time of the associated CME onset was given by Harrison et al. (1985), Harrison (1986). The precursor activity was found in close spatial 
relationship to the area of the CME origin. Dere et al. (1997a) were the first to report on-disk observations of a CME onset using images taken by the Extreme-ultraviolet Imaging Telescope (EIT) aboard the Solar and Heliospheric Observatory (SoHO). From this analysis, it was inferred that the CME is triggered in a small volume associated with an initial small EUV brightening at one end of the erupting filament.

It is generally believed that filaments consist of cool gas (of chromospheric temperature) supported in the corona by largescale curved and twisted magnetic flux tubes (e.g. Priest et al. 1989). Filaments become useful in tracking the evolution of the large-scale field system by following their eruption. Detailed analyses of the geometry and evolution of flare-associated filament eruptions can provide a tool for investigating the eruption destabilisation and relationships between the associated phenomena. The "fast-rise" of filament acceleration and the impulsive flare phase are often preceded by a filament "slow-rise" with little or no acceleration at $<10 \mathrm{~km} \mathrm{~s}^{-1}$ (e.g. Moore \& Sterling 2006; Chifor et al. 2006; Isobe \& Tripathi 2006; Isobe et al. 2007). Several studies have found preflare activity such as small X-ray and EUV brightenings at the onset of the filament's slowrise (e.g. Sterling \& Moore 2005; Chifor et al. 2006).

To date there are several ideas for the initiation of solar eruptions; see e.g. Moore \& Sterling (2006) for a recent review. These include internal tether-cutting (see e.g. Moore \& Roumeliotis 1992), external tether-cutting or "breakout" (Antiochos 1998; Antiochos et al. 1999), or the flux cancelation model originally proposed by van Ballegooijen \& Martens (1989) and further investigated by Linker et al. (2003). Other models of filament eruptions include the kink instability of the filament/flux rope (Fan 2005; Rust \& LaBonte 2005; Török \& Kliem 2005). These models can be distinguished from each other mainly during the eruption initiation; they all tend to look the same during the eruption impulsive phase. Conclusive spatial and temporal relations between of the preflare activity and the magnetic topology of the erupting system could provide critical observational evidence for the triggers predicted by the different models. Section 2 outlines the models which we have examined in this study and the essential observable differences.

Although the preflare activity has been studied extensively in the past, most of the analyses remained inconclusive, mainly due to a lack of high-resolution data, both spatial and temporal. At present, the best spatial and temporal resolution data are provided by the Transition Region and Coronal Explorer (TRACE) in EUV and the Reuven Ramaty High Energy Solar Spectroscopic Imager (RHESSI) in X-rays.

Recently, Chifor et al. (2006) made use of TRACE and RHESSI data to analyse the early phases of a filament eruption observed at the solar limb. The eruption was seen in the EUV $171 \AA$ filters of both $S o H O / E I T$ and TRACE, with complementary X-ray data from RHESSI and microwave images from the Nobeyama Radioheliograph (NoRH). The filament activation was first observed beneath one anchored footpoint where a localised preflare X-ray brightening was reported to be temporally and spatially correlated with the onset of the filament slow-rise. It was concluded that localised reconnection events took place at one end of the filament, eventually destabilising the entire system, which entered a fast-rise phase. However, while this event provided excellent multiwavelength observations of the initiation and evolution of the eruption, there was no information about the photospheric magnetic field configuration since the filament was situated near the limb. Chifor et al. (2006) showed that both plasma heating and electron acceleration occurred at one footpoint of the erupting filament, simultaneous with the slow-rise onset. Based on both the location (beneath the filament) and timing of the preflare X-ray source, as well as the filament slow-rise and fast-rise phases being consistent with the tether-cutting runaway reconnection scenario, they suggested that internal tether-cutting was a possible eruption trigger. However, it is difficult to validate this result without a better understanding of the magnetic field topology.

In this paper, we investigate the presence of preflare brightenings and their potential for providing information about the the physical mechanisms triggering the eruption. Models for the eruption mechanisms are described in Sect. 2. We analyse two on-disk filament eruptions which have the advantage that the geometry and evolution of the magnetic fields can be determined from magnetograms. X-ray spectral and imaging data from RHESSI are combined with images from TRACE at $195 \AA$, while the photospheric magnetic field is observed in magnetograms taken by the Michelson Doppler Imager (MDI) instrument on $\mathrm{SoHO}$ (the different instruments are described in Sect. 3). Additionally, we sample six active region filament eruptions which have been reported in the literature but have not been investigated in the context of the X-ray preflare emission (the analysis of all 8 events is given in Sect. 4). We focus on the preflare activity of these events and summarise our results in Sect. 5. The spatial and temporal relationships between the preflare brightenings and the motion of the erupting filament (and the magnetic field topology, for on-disk events) provide valuable clues about the eruption mechanisms. In Sect. 6 we discuss the implications of these signatures for eruption models.

\section{Eruption models}

In our analysis we considered three basic eruption models which have been recently discussed by Moore \& Sterling (2006): internal tether-cutting, external tether-cutting, and an ideal MHD (e.g. kink) instability. Each of the three proposed triggers can be described for the case when the erupting filament sustained by a sheared core field is in the central lobe of a quadrupolar magnetic field configuration. Moore \& Sterling (2006) sketch the eruption initiation in each of the three cases in a sequence of cartoons which is reproduced in Fig. 1. It is noted that the internal tethercutting reconnection and ideal MHD instability can also occur in the case when the sheared field is the core of an isolated bipole, but external tether-cutting (breakout) reconnection can strictly occur only in quadrupolar configurations.

If reconnection takes place, it is expected to begin either below the erupting filament, producing brightenings in the sheared core field, or at the magnetic null point above the erupting central lobe, producing remote brightenings. The terminology used by Moore \& Sterling (2006) is "slow-runaway reconnection" for reconnection during the slow-rise phase and "explosive reconnection" for reconnection during the fast-rise phase of the eruption.

We compare our observed early eruption activity with the signatures predicted by these models as suggested by Fig. 1. In particular, internal-tether cutting requires brightenings low in the core field, i.e. close to the magnetic polarity inversion line (PIL), during the slow-rise phase onset. External tether-cutting predicts brightenings before the onset of the filament eruption, far from the core field. An ideal MHD trigger would show an erupting filament entering the fast-rise phase immediately, with no precursor or brightening anywhere in the eruptive region prior to the main phase. 




Fig. 1. Schematic representation of the 3 trigger mechanisms after Moore \& Sterling (2006). A canonical quadrupolar magnetic field configuration was chosen for each of the three cases: internal tether-cutting (left panels), external tether-cutting (middle panels), and ideal MHD instability (right panels). In each scenario, the magnetic field is shown prior to eruption (top), just after eruption onset (middle) and when the eruption is well under-way (bottom). The sites of the precursor and main phase emissions (observed respectively as preflare brightenings and flare brightenings during the impulsive phase of the eruption) are indicated.

\section{Observations}

We have scanned the online collection of TRACE movies ${ }^{1}$ and selected two on-disk filament eruptions with sufficient coverage of the early eruption phases from both TRACE and RHESSI (events 1 and 2 listed in Table 1). The sampling criteria for these events may be summarised as follows:

1. The event is an active region filament eruption accompanied by a flare.

2. The event is on-disk (longitude within the range $\pm 65^{\circ}$ ).

3. There is TRACE coverage throughout the filament eruption.

4. There is RHESSI coverage for the preflare phases of the accompanying flare, starting at least $5 \mathrm{~min}$ before the impulsive phase onset.

A further six events (indexed 3 to 8 of Table 1) were selected from observations of filament eruptions reported in the literature. The criteria for selecting these additional events were as above, except for the second (limb events were also included) and fourth (there is RHESSI coverage only after launch in February 2002).

The TRACE instrument (Handy et al. 1999) consists of a $30 \mathrm{~cm}$ aperture Cassegrain telescope observing the solar corona in three coronal EUV wavelengths (Fe IX/X, $171 \AA$; Fe XII/ XXIV, $195 \AA$; Fe XV, $284 \AA$ ), as well as in H I Lyman- $\alpha$ (1216 ̊), C IV (1550 ̊), UV continuum (1600 ̊), and white light $(5000 \AA)$. Its unprecedented high-spatial resolution (of approximately $1^{\prime \prime}$ ) and cadence (as low as 20 s) render this instrument suitable for observing eruptive events in detail. Fe IX and Fe $\mathrm{X}$ lines dominate the emission in the $171 \AA$ TRACE filter while Fe XII usually dominates the emission in the $195 \AA$ filter, making these passbands sensitive to temperatures of 1-2 MK. However, Phillips et al. (2005) have shown that the $171 \AA$ filter also has a high-temperature response $(\sim 10-20 \mathrm{MK})$ due to the continuum and Fe XX contributions. During solar flares it has also been found that the TRACE $195 \AA$ channel can contain some Fe XXIV emission (e.g. Phillips et al. 2005; Tripathi et al. $2006 \mathrm{~b})$ so that high-temperature features $(\sim 10-20 \mathrm{MK})$ present in RHESSI X-ray images are also detectable in the TRACE EUV channels.

We processed the raw TRACE data by means of standard SolarSoft routines, subtracting the image pedestal and dark current as well as correcting for radiation spikes. Much of our

\footnotetext{
1 At http://trace.lmsal.com/POD/TRACEpod.html
}

analysis involved the co-alignment of images from TRACE with images from RHESSI and MDI. This is not a straightforward task because the TRACE absolute pointing is uncertain at up to the $40^{\prime \prime}$ level. All analysed TRACE images, therefore, required a pointing correction, cross-checked at different times during the events. Full-disk EUV images taken by SoHO/EIT (Delaboudinière et al. 1995) proved useful for feature coalignment in a similar wavelength band using the methods described in Gallagher et al. (2002). Alternatively, when EIT images were missing, we co-aligned simultaneous TRACE 1600 images with $S o H O / M D I$ images, and then used the resulting shift values to correct simultaneous TRACE EUV images. In event 1 of Table 1, we complemented TRACE observations with blue wing $\mathrm{H} \alpha(-0.6 \AA)$ images of the filament eruption obtained at the Big Bear Solar Observatory (BBSO), with 1" pixel size and 1 min cadence.

We made use of X-ray light-curves from the Geostationary Operational Environmental Satellites (GOES). These monitor the spatially-integrated solar emission in two broadband SXR filters (0.5-4 and 1-8 $\AA$ ). Spatial information about the X-ray preflare brightenings and flare evolution was provided by RHESSI. RHESSI consists of rotation modulation collimators situated in front of a spectrometer with nine high-resolution germanium detectors (Lin et al. 2002). Providing a spatial resolution as low as $\sim 2^{\prime \prime}$ and X-ray spectral resolution of $\sim 1 \mathrm{keV}$, RHESSI is sensitive to solar high-energy emission from $3 \mathrm{keV}$ to $17 \mathrm{MeV}$. We obtained X-ray images using the CLEAN (Hurford et al. 2002) algorithm available in the standard RHESSI software, using data from detectors 1-9 with natural weighting and "local average" enabled.

For the sampled on-disk events, we obtained line-ofsight photospheric magnetic field observations taken with the SoHO/MDI instrument (Scherrer et al. 1995). MDI measures the line-of-sight, i.e. the longitudinal component of the magnetic field with a resolution of $2^{\prime \prime}$ from the Zeeman splitting of the Ni I (6768 ̊) line.

\section{Data analysis and results}

In the next two subsections we present the two events analysed here in detail (events 1 and 2 of Table 1), followed by descriptions of the 6 revisited filament eruptions (events 3 to 8 of Table 1). 


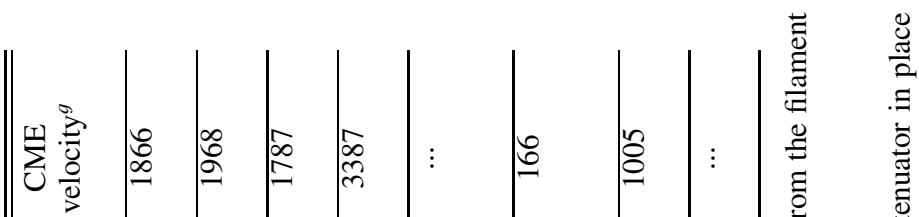

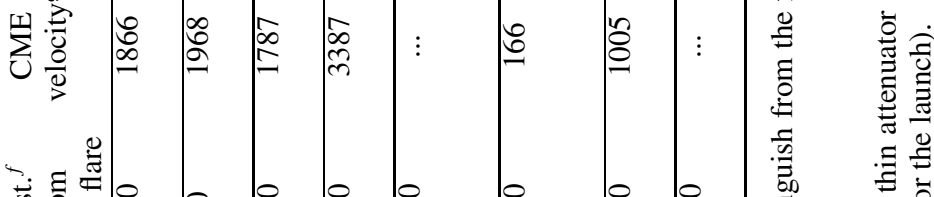



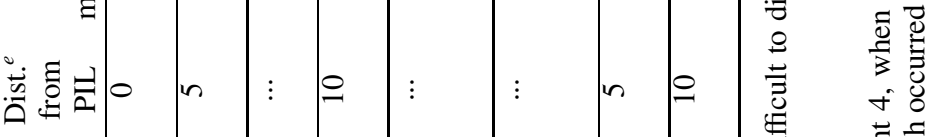

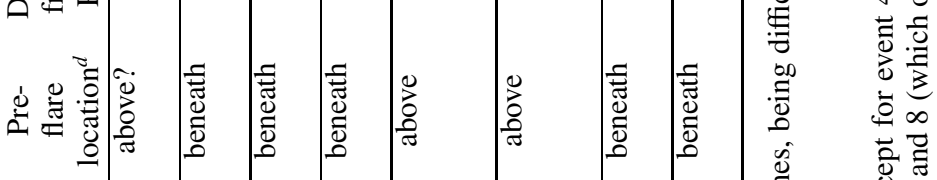

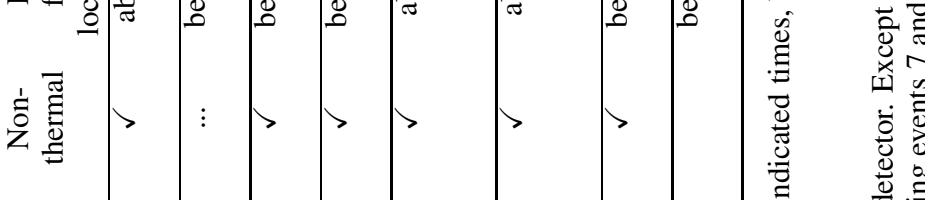



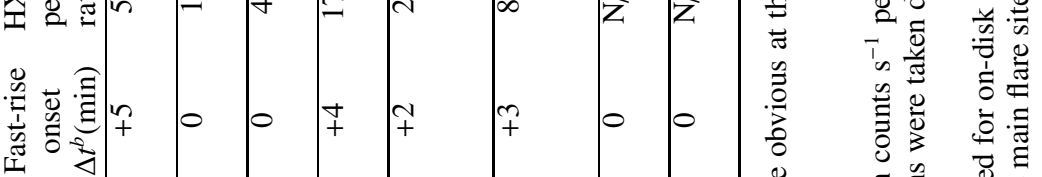

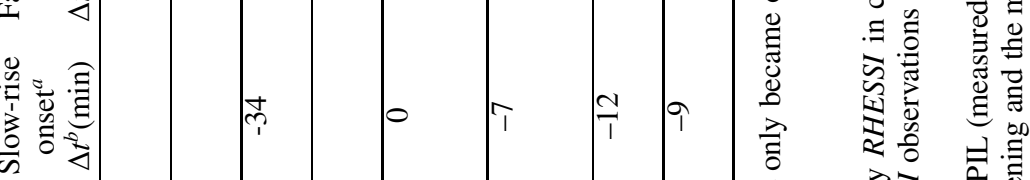

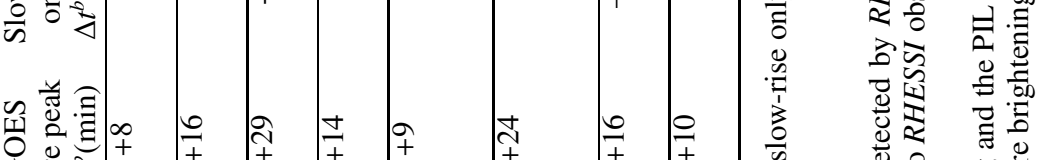

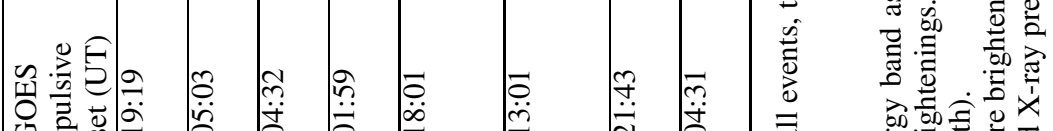

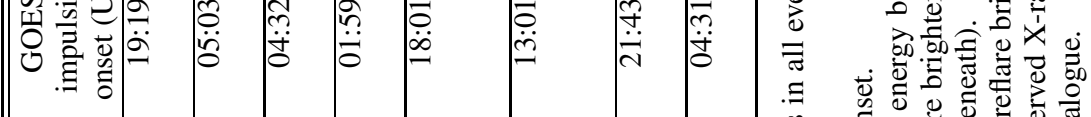

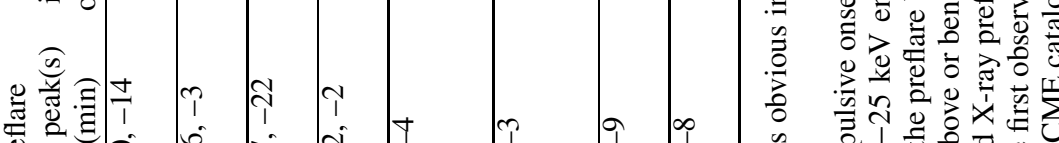





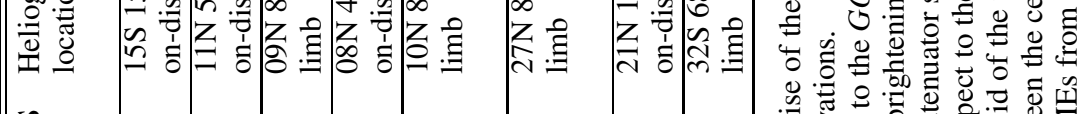

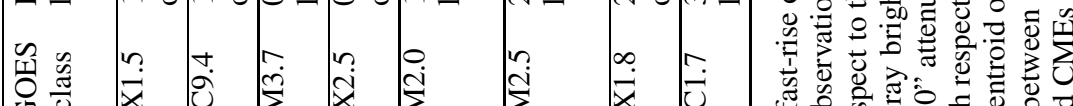

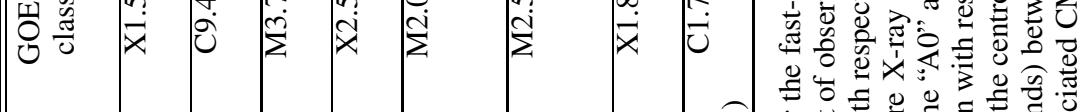

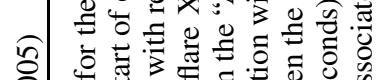

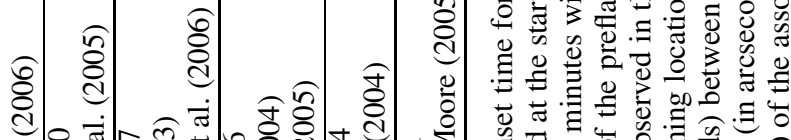



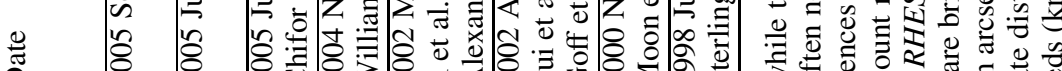

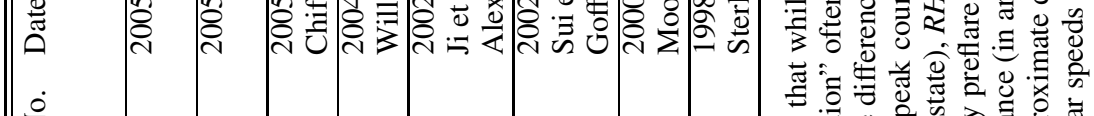

ib

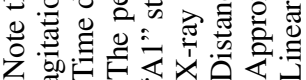



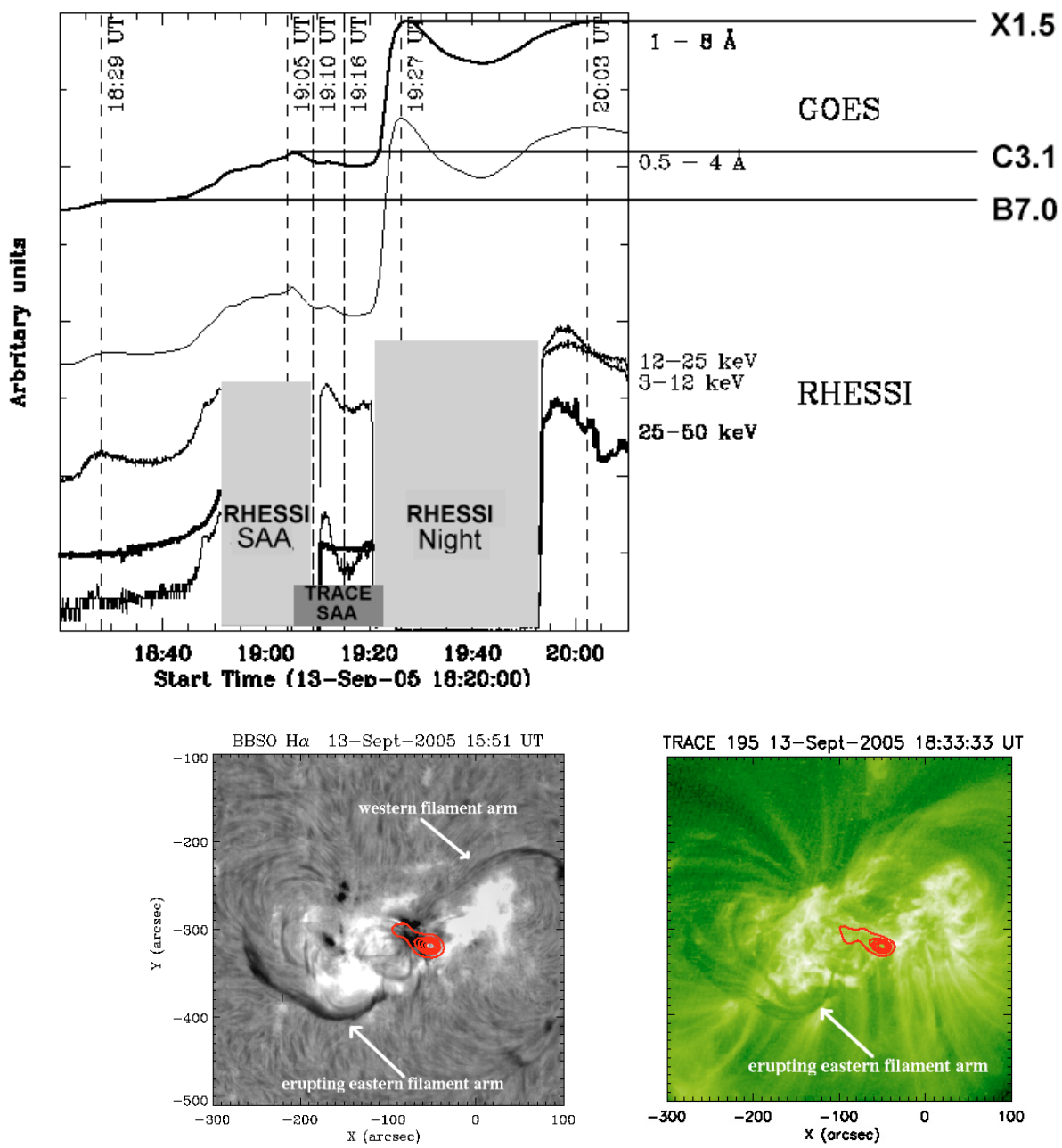

Fig. 2. GOES $0.5-4 \AA$ (thin curve) and $1-8 \AA$ (thick curve) light-curves during the 2005 Sep. 13 event (event 1 of Table 1). RHESSI count rates in the energy ranges of $3-12 \mathrm{keV}, 12-25 \mathrm{keV}$, and $25-50 \mathrm{keV}$ were averaged over RHESSI detectors 1, 3, 4, 5, 6, 8 and 9. The periods during which RHESSI entered the SAA (18:51-19:09 UT) and then night-time (19:21-19:52 UT) are marked by light grey areas, and the TRACE SAA period (19:07-19:22 UT) is marked by the dark grey area. Prior to night-time, RHESSI observed with no attenuators in front of its detectors. When RHESSI emerged from night-time ( 19:53 UT), both its thin and thick attenuators were inserted ("A3" state). The y axis has a logarithmic scale. Indicated on the plot are the times of the two preflare brightening peaks in the GOES 0.5-4 $\AA$ at 18:29 and 19:05 UT.

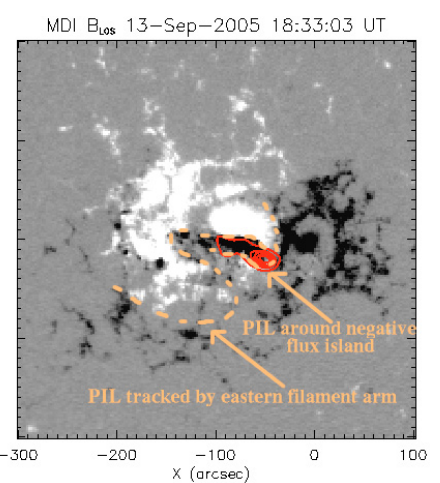

Fig. 3. Left panel: $\mathrm{H} \alpha$ observation of the filament some $3 \mathrm{~h}$ prior to eruption on 2005 Sep. 13 (event 1 of Table 1). The image was taken at BBSO. The filament is seen in absorption as a dark S-shaped feature. The eastern and western arms of the S-shape are marked on the figure. Only the eastern arm was observed to erupt. The first SXR preflare brightenings (6-12 keV, red contours) were observed by RHESSI close to the centre of the S-shape between 18:25 UT and 18:51 UT. Middle panel: the S-shaped filament seen as an absorption feature in TRACE $195 \AA$ Anly the eastern (erupting) arm is visible in EUV images. Simultaneous RHESSI preflare brightening contours are also overlaid. This image forms part of video1. Right panel: MDI magnetogram taken prior to eruption at 18:33 UT with simultaneous RHESSI preflare contours. The PIL has a complex shape marked by the orange dashed curve. There are two major components of the observed PIL: one loop around the island of negative flux in the centre of the active region, and one eastern arm tracked by the eastern filament arm.

\subsection{September 13 (event 1: on-disk)}

The filament eruption on 2005 September 13 occurred at the heliographic location $15 \mathrm{~S} 15 \mathrm{E}$ and was associated with a GOES X1.5 class flare peaking at 19:27 UT (Fig. 2). Subsequently, a $\mathrm{CME}$ was detected by the $\mathrm{SoHO} /$ Large Angle and Spectrometric Coronograph (LASCO)/C2 around 20:00 UT with a speed of $1866 \mathrm{~km} \mathrm{~s}^{-1}$ according to the LASCO CME catalogue ${ }^{2}$. A stationary on-disk filament was observed at 15:51 UT in $\mathrm{H} \alpha$ as a dark S-shape (Fig. 3, left panel), approximately $3 \mathrm{~h}$ prior to its eruption at $\sim 19: 24$ UT. Other studies of this filament eruption have been carried out independently by Nagashima et al. (2007) and Wang et al. (2007).

We observed the activation of the filament mainly in TRACE images taken in the $195 \AA$ passband (Fig. 3, middle panel). Only the eastern arm of the filament appeared dark in EUV images. Since no EIT data were available at the time of the eruption, we obtained the TRACE pointing correction values by coaligning images taken by TRACE at $1600 \AA$ with simultaneous

\footnotetext{
${ }^{2}$ http://cdaw.gsfc.nasa.gov
}

MDI magnetograms. RHESSI preflare images were obtained in the $6-12 \mathrm{keV}$ band between $\sim 18: 25$ UT and 18:51 UT, when the spacecraft entered the South Atlantic Anomaly (SAA). These images show preflare brightenings situated close to the centre of the S-shaped filament at the location $x=-50^{\prime \prime}$ and $y=-320^{\prime \prime}$ (Fig. 3, middle panel), where $x$ and $y$ are the coordinates measured from the centre of the visible solar disk with an estimated uncertainty of $\pm 3^{\prime \prime}$. MDI magnetograms with overlaid RHESSI contours revealed these preflare brightenings on the PIL surrounding an island of negative polarity in the middle of the active region (Fig. 3, right panel). This loop component of the PIL has its eastern end connected to the neighbouring PIL traced by the eastern arm of the filament.

The beginning of the activity is marked by two weak, distinct, enhancements in the SXR GOES light-curves (between $\sim 18: 25$ UT and 19:20 UT) which peak at 18:29 UT and 19:05 UT, at the B7 and C3 levels, respectively (Fig. 2). The destabilisation of the system became apparent at the time of the X-ray preflare emission peak (19:05 UT), when the entire region strongly brightened in the TRACE EUV images. TRACE was in the SAA for approximately $15 \min (19: 07-19: 22$ UT) 



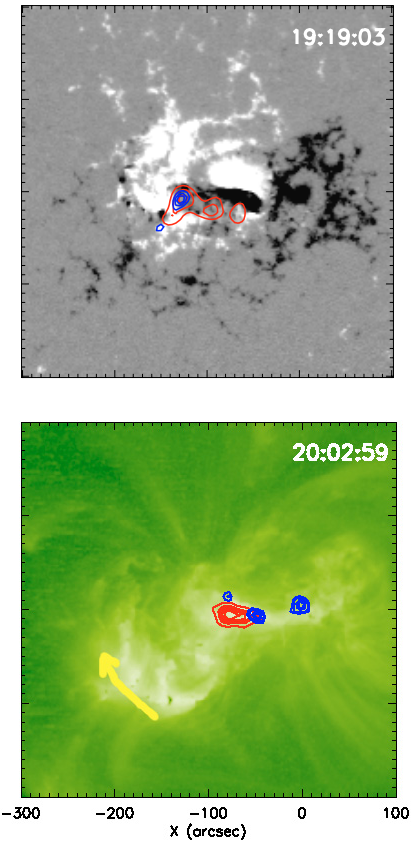

Fig. 4. Evolution of the filament eruption on 2005 Sep. 13 (event 1 of Table 1). Top row (19:10-19:20 UT): MDI maps with nearly simultaneous RHESSI 6-12 keV (red) and 12-25 keV (blue) contours. All three MDI maps form part of video2. The preflare X-ray brightenings have the apparent form of a loop-top source with two footpoints traveling rapidly from west to east (right to left) along the component of the PIL bounding the south side of the negative polarity island, as indicated by the yellow arrow. There is no TRACE data available between 19:07-19:22 UT because the instrument crosses the SAA. Bottom row (19:30-20:03 UT): rising from the preflare X-ray brightening initial location, the left half of the filament erupts asymmetrically (white arrows indicate its motion). The location of the initially anchored footpoint (FP) of the filament observed to rise from 19:24 UT is indicated with the yellow arrow in the left image (taken at 19:29 UT). Post eruption EUV brightenings propagate along and separate away from the PIL, marking the footpoint regions which track the projection of the erupted filament in the direction indicated by the yellow arrows. RHESSI 6-12 keV (red) and 30-40 keV (blue) X-ray emission during the main energy release phase (flare impulsive phase) is overlayed on the image taken at 20:03 UT (right panel). All TRACE maps form part of video1.

but the event could still be monitored with the RHESSI X-ray images and the BBSO $\mathrm{H} \alpha$ images. We overlayed 6-12 keV and $12-25 \mathrm{keV}$ contours on co-temporal MDI magnetograms in Fig. 4, top row.

We analysed the X-ray spectrum of the preflare brightening observed with RHESSI by means of imaging spectroscopy with the Object Spectral Executive (OSPEX), which uses version 5.2 of the CHIANTI (Dere et al. 1997b; Landi et al. 2006) atomic code. The thermal plasma emission was modeled with an isothermal component (describing the free-free and free-bound continuum) and one Gaussian function to fit the $6.7 \mathrm{keV} \mathrm{Fe}$ line feature (method described in detail by Phillips et al. 2006). The observed high-energy emission $(\gtrsim 10 \mathrm{keV})$ was modeled as a nonthermal component with a power-law distribution $I(E) \propto$ $E^{-\gamma}$, where $\gamma$ represents the power-law index of the photon flux spectrum. The power-law distribution breaks to $I(E) \propto E^{-1.5}$ at energies $<10.5 \mathrm{keV}$. An example of a RHESSI spectrum at 19:11 UT fitted to the sum of these three components is given in Fig. 5. The fitting suggests that, at energies above $\sim 10 \mathrm{keV}$, the nonthermal contribution dominated over the thermal component.

The X-ray brightenings observed in the 6-12 keV and 12-25 keV RHESSI images traveled rapidly (with a velocity of approximately $156 \mathrm{~km} \mathrm{~s}^{-1}$ ) from west to east (right to left) along the PIL component bounding the southern side of the negative polarity island, in the same direction as the erupting filament arm (Fig. 4). To the best of our knowledge, this is the first observation of such evolution in the pre-eruption X-ray activity. The $\mathrm{X}$-ray preflare brightenings had the apparent form of a loop-top source (seen in both 6-12 keV and 12-25 keV images) and two footpoints (observed in the 6-12 keV energy band). However, this was difficult to determine with certainty due to projection effects. It is possible that this motion is tracking the propagation of magnetic reconnection along the PIL, in agreement with the $3 \mathrm{D}$ propagation of magnetic reconnection during asymmetric filament eruptions discussed by Grigis \& Benz (2005).

We note that possibly because the filament was on-disk, we were not able to observe a slow-rise of the filament prior to its fast-rise commencing at $\sim 19: 24$ UT. At this time, the filament (observed in BBSO $\mathrm{H} \alpha$ and TRACE EUV images) was seen to rise at a speed $>100 \mathrm{~km} \mathrm{~s}^{-1}$, well above the slow-rise speed of $<10 \mathrm{~km} \mathrm{~s}^{-1}$ generally reported at the beginning of filament eruptions. The eastern half of the filament erupted asymmetrically from west to east (right to left) as shown in the TRACE images of Fig. 4, bottom row. The site of the initially anchored footpoint (FP) that was first observed to rise is indicated in Fig. 4, bottom row, left panel, at the approximate location $x=-120^{\prime \prime}$ and $y=$ $-350^{\prime \prime}$. The rising footpoint at $\sim 19: 24$ UT appeared co-spatial with the X-ray preflare brightenings observed with RHESSI at 19:21 UT, just before the instrument entered night-time.

Only the eastern arm of the S-shaped filament was observed to erupt. During its eruption, EUV brightenings propagated in the direction of motion along the PIL under the eastern filament arm, separating at the same time away from the PIL. This simultaneous propagation along and away from the PIL of posteruption brightenings in EUV during asymmetric filament eruptions has been studied by Tripathi et al. (2006a) (Fig. 6). The site of the main energy release was observed close to (within $\sim 20^{\prime \prime}$ of) the initial preflare location (Fig. 4, bottom row, right panel). 


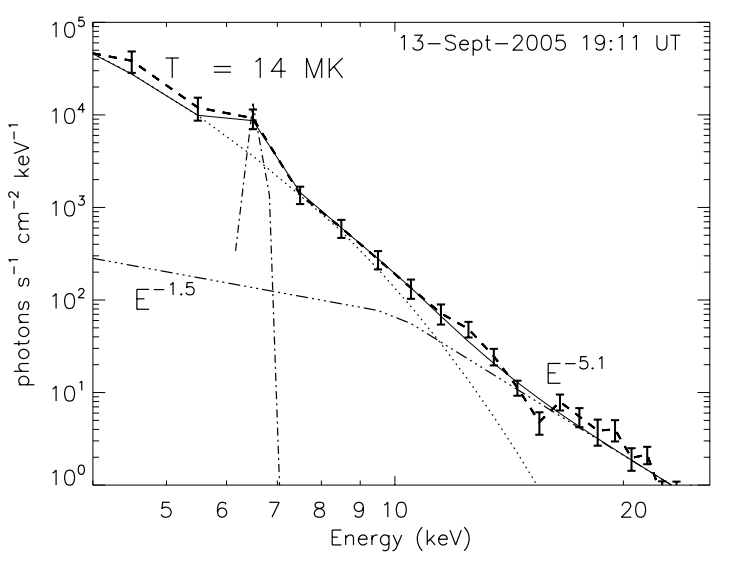

Fig. 5. RHESSI spectrum (dashed curve, with error bars) of the preflare brightenings accumulated for $80 \mathrm{~s}$ around 19:11 UT on 2005 Sep. 13 (event 1 of Table 1). The thermal continuum was modeled with one isothermal component (dotted curve) using CHIANTI v.5.2. One Gaussian function was added to fit the Fe line feature observed by RHESSI $\sim 6.7 \mathrm{keV}$ (dashed-dot curve). The high-energy tail of the spectrum was fitted with a power-law component $E^{-5.1}$ (dashed dot-dot-dot) breaking to $E^{-1.5}$ at energies below $10.5 \mathrm{keV}$. The sum of all three spectral components (best-fit model) is represented by the continuous curve. The fitting range was $5-25 \mathrm{keV}$.

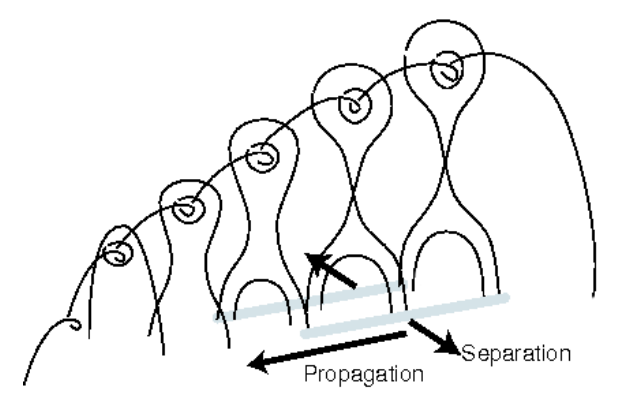

Fig. 6. 3D schematic diagram of an asymmetric filament eruption such as the erupting filament on 2005 Sep. 13 (event 1 of Table 1). The direction of EUV brightening propagation along and separation away from the PIL is marked by arrows. This figure was adapted from Tripathi et al. (2006a).

To determine the topology of the magnetic field in the erupting region, we investigated a sequence of MDI images before, during, and after the event. We detect a small parasitic positive polarity emerging in the strong negative-flux region, at a location close to the site of the SXR preflare brightenings (Fig. 7). A faint parasitic polarity could be noticed as early as $19 \mathrm{~h}$ (at 00:03 UT) prior to the filament eruption. The small patch of positive polarity was still faint at 19:22 UT, attaining a maximum in the next available image at 19:26 UT (close to the flare peak), and then decayed until it disappeared at about 20:40 UT. The observed emerging flux may provide a potential cause for the eruption (Subramanian \& Dere 2001).

\subsection{July 30 (event 2: on-disk)}

The event of 2005 July 30 (11N 51E) consisted of a double filament eruption accompanied by a double-flare peaking at 05:19 UT (GOES class C9.4) and 06:34 UT (GOES class X1.3), respectively (Fig. 8). We focused on the filament eruption associated with the first flare, which commenced at 05:03 UT, and its associated preflare activity. Prior to its eruption, an S-shaped filament was observed in absorption in TRACE EUV $171 \AA$

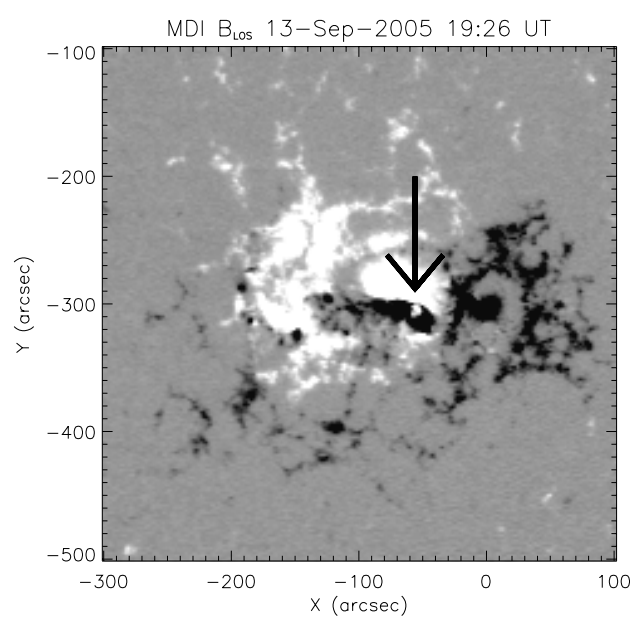

Fig. 7. MDI magnetogram taken before close to the peak of the 2005 Sep. 13 flare (event 1 of Table 1). The small patch of positive magnetic flux which becomes prominent close to the flare peak at 19:26 UT. This magnetogram forms part of video 2 .

images (Fig. 9). TRACE observed the onset and evolution of the filament eruption for approximately $6 \mathrm{~h}$ between 03:00-09:00 UT. The pointing offset was obtained by means of co-alignment with $\mathrm{SoHO} / \mathrm{EIT} 171 \AA$ A images.

We observed the northern part of the filament being activated as early as 03:01 UT, with transient EUV brightenings accompanied by slow motion that resulted in a net upward displacement. The EUV brightenings have corresponding small enhancements in the GOES and RHESSI X-ray light-curves. In particular, we noticed two small X-ray bursts in the higher-energy (0.5-4 $\AA$ ) GOES light-curves at 04:54 and 05:00 UT (Fig. 8), just before the filament eruption at 05:03 UT. RHESSI light-curves show a burst in the 3-12 keV light-curves at 05:02 UT, and an impulsive spike in the 12-25 keV light-curves occurring at 05:04 UT. At 05:01 UT, the preflare brightenings in EUV appeared to travel between the two end footpoints of the S-shaped filament. We obtained RHESSI SXR images (6-12 keV) after 05:01 UT. These preflare X-ray contours were found spatially coincident with the EUV brightenings which appeared first at the footpoint of the northern filament part $\left(x=-850^{\prime \prime}\right.$ and $\left.y=120^{\prime \prime}\right)$ at 05:02 UT, and then at the footpoint of the southern filament part $\left(x=-750^{\prime \prime}\right.$ and $y=120^{\prime \prime}$ ) at 05:03 UT, as shown in Fig. 9 (top row, left and middle panels). The evolution of the photospheric magnetic field for this event suggests that the location of the preflare X-ray brightenings corresponded to regions of canceling magnetic field possibly driven by emerging flux (Fig. 10). At approximately 05:03 UT the southern part of the filament erupted rapidly, starting from the location of the X-ray preflare brightening close to its initially anchored footpoint marked in Fig. 9 (top row, middle panel). The direction of the filament eruption is marked with an arrow in Fig. 9 (middle row, left panel). The erupting material displayed a writhing evolution before sliding back down. We note that we did not observe a slow-rise prior to the impulsive eruption of this part of the filament. However, this structure was closely linked to the northern part of the filament for which a slow-rise was visible from the beginning of the TRACE observations approximately $2 \mathrm{~h}$ prior to the eruption fast-rise phase. These observations are suggestive of an internal tether-cutting mechanism driving the eruption of the southern filament.

Around 05:55 UT this eruption reached the middle of the filament channel. Simultaneously, the northern part of the filament entered its fast-rise phase, starting from its anchored footpoint 


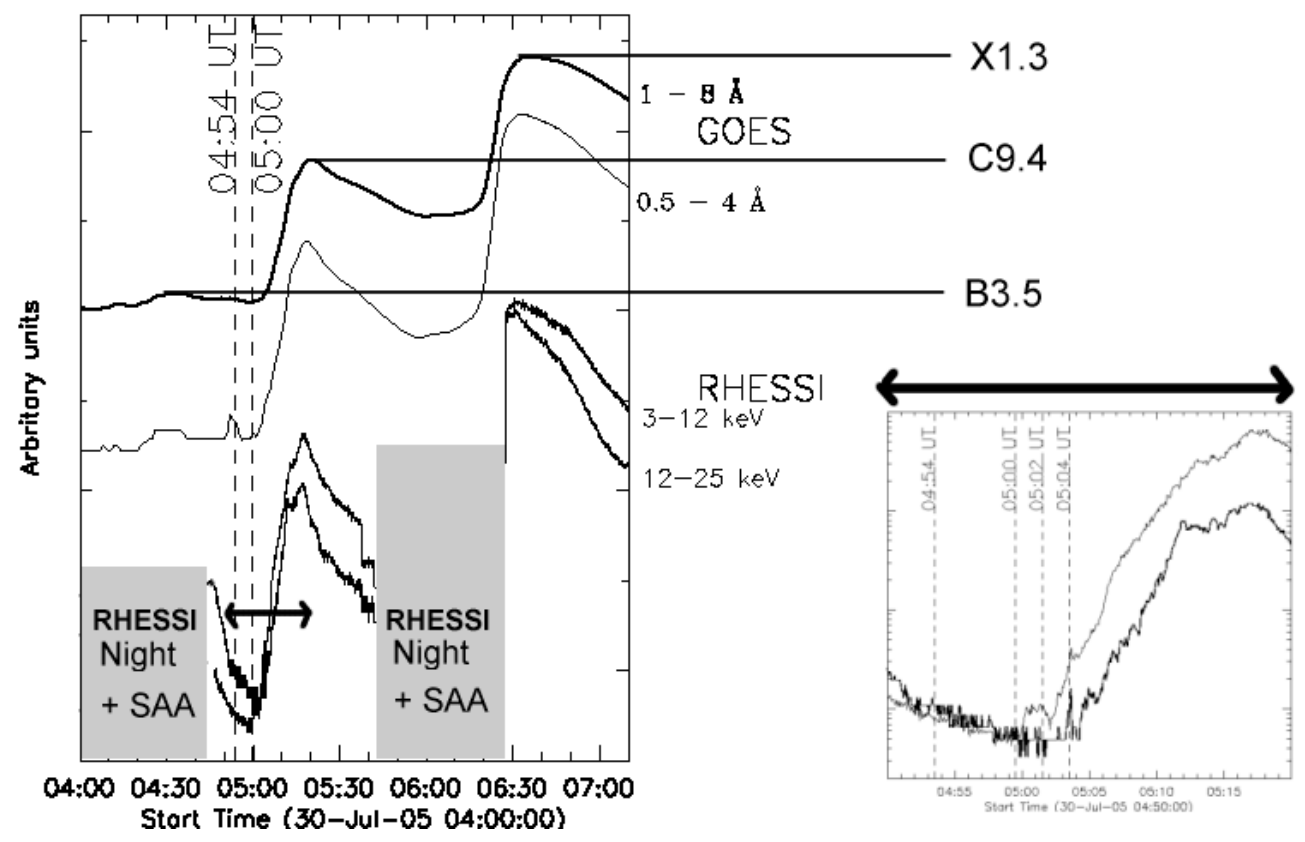

Fig. 8. Left panel: GOES $0.5-4 \AA$ (thin curve) and 1-8 $\AA$ (thick curve) light-curve profiles during the 2005 July 30 filament eruption (event 2 of Table 1). The preflare brightenings at 04:54 and 05:00 UT in the higher energy channel (0.5-4 $\mathrm{A})$ are indicated. RHESSI count rates in the energy ranges of 3-12 keV and 12-25 keV, averaged over detectors $1,3,4,5,6,8$ and 9 are also plotted. The $y$ axis has a logarithmic scale. The RHESSI spacecraft emerged from a period of night-time and SAA (marked by the grey areas) at 04:46 UT. The double arrow between 04:50-05:20 UT marks the time interval over which the RHESSI light-curves are plotted separately in the right panel, in order to distinguish the transient preflare $\mathrm{X}$-ray bursts around the time of the impuslive flare onset at 05:03 UT. Right panel: RHESSI count rates in the energy ranges of 3-12 keV and $12-25 \mathrm{keV}$, averaged over detectors $1,3,4,5,6,8$ and 9. The preflare activity in the form of relatively weak SXR bursts is conspicuous between approximately 04:54 UT and 05:04 UT. We note, in particular the enhancement in the 3-12 keV light-curves at 05:02 UT, and the impulsive spike in the 12-25 keV light-curves at 05:04 UT.
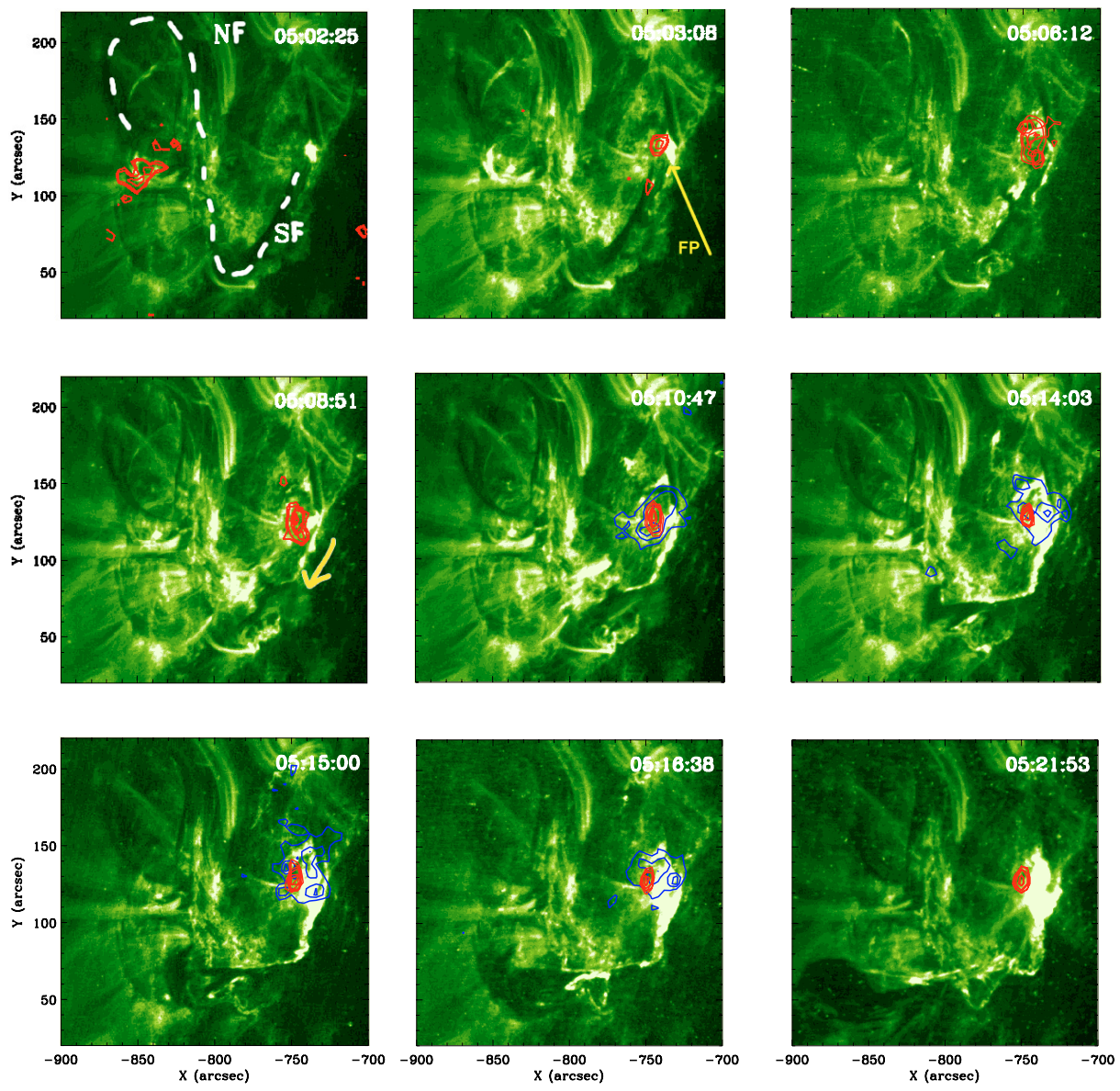

Fig. 9. TRACE $171 \AA$ images showing the 2005 July 30 filament eruption (event 2 of Table 1). RHESSI 6-12 keV and 12-25 keV contours are overplotted in red and blue, respectively. The northern (NF) and southern (SF) parts of the S-shaped filament are marked (top row, first image). The erupting footpoint (FP) of the southern filament, anchored prior to its fast-rise at 05:03 UT, is indicated on the image taken around this time (first row, middle panel). The direction of the southern filament eruption and EUV propagation is indicated by the yellow arrow on the TRACE image at 05:08:51 UT (middle row, left panel). All panels form part of video3. 




Fig. 10. MDI magnetograms taken prior to (left panel), just before (middle panel) and after (right panel) the filament eruption on 2005 July 30 (event 2 of Table 1). RHESSI 6-12 keV contours taken at 05:02:00 UT (red) and 05:03:08 UT (yellow) are overlayed. These SXR preflare brightenings coincide with regions of evolving magnetic features (encircled in the images prior to and after the eruption) which suggest magnetic flux cancelation possibly driven by emergence of new flux.

in the middle of the S-shape. The northern filament eruption was accompanied by the second (more intense) flare.

During the southern filament eruption between 05:0305:55 UT, we observed the propagation of post-eruption EUV brightenings as expected from the extension of "standard" 2D flare models to 3D (e.g. Tripathi et al. 2006a). However, the RHESSI HXR sources remained at the same locations. The site of the HXR (12-25 keV) emission is shown in Fig. 9, coincident with the position of the preflare brightenings (at the approximate coordinates $x=-750^{\prime \prime}$ and $y=120^{\prime \prime}$ ).

We note that while the HXR emission was observed as relatively short bursts, mainly during the flare impulsive phase of the flare, the EUV emission was present throughout the entire event, tracking the complete evolution of the eruption past its impulsive phase. Therefore, studying the evolution of an eruptive event requires multiwavelength observations which combine context EUV images throughout the entire event with highcadence $\mathrm{X}$-ray observations.

\subsection{July 27 (event 3: limb)}

A spectacular filament eruption occurred over the eastern solar limb (09N 89E) on 2005 July 27 (Fig. A.1). This event has been described in detail by Chifor et al. (2006), using TRACE $171 \AA$ images and RHESSI X-ray images complemented by microwave observations from NoRH. The prominence erupted such that its southern connected end started to rise slowly noticeably in correlation with the appearance of a discrete, localised X-ray preflare brightenings, peaking at 03:54 UT. The preflare brightening source was situated close to $\left(\sim 20^{\prime \prime}\right.$ south of $)$ the visible barb and below the erupting footpoint (Fig. A.1, middle panel). From RHESSI spectral analysis of the X-ray preflare enhancement, Chifor et al. (2006) found evidence of thermal X-ray emission at $\sim 15 \mathrm{MK}$, and nonthermal bremmstrahlung emission at energies above $10 \mathrm{keV}$, suggesting localised low magnetic reconnection beneath the filament. These observations were suggestive of the eruption being triggered by internal-tether cutting reconnection. The site of the later main energy release can be observed during the fast-rise phase of the filament eruption approximately $50^{\prime \prime}$ north of the preflare location (Fig. A.1, bottom panel).

\subsection{November 10 (event 4: on-disk)}

The event on 2004 November 10 (08N 49W) has been reported previously by Williams et al. (2005), who observed a highly-localised preflare brightening in TRACE $1600 \AA$ images at 01:52 UT, $\sim 7 \mathrm{~min}$ prior to the flare onset (Fig. A.2). This brightening was co-spatial with the emergence of a small magnetic bipole near the PIL. Additionally, we find a preflare X-ray enhancement in GOES light-curves, with peaks at 01:47 UT and 01:57 UT. We also observed corresponding enhancements in X-ray RHESSI light-curves. We imaged the preflare enhancement around $\sim 01: 58$ UT in the RHESSI 6-12 keV wavelength band, and found the source situated near the PIL (within 10"), close to the approximate location from which the filament was first observed to rise (Fig. A.2, top right and bottom left panels).

We observed a fast-rise phase of the filament already on its way by 02:03 UT, after the onset of the impulsive flare phase. There are no TRACE images in the $1600 \AA$ available before 02:03 UT to distinguish the onset time of a potential initial slowrise of the filament. The site of the main flare emission (Fig. A.2, bottom right panel) is observed along the PIL, within $30^{\prime \prime}$ of the preflare emission.

Williams et al. (2005) found that an exponential function fitted the height-time profile of the erupting filament, suggesting an MHD kink instability trigger. They argue that the filament prior to the eruption was highly twisted, which would also point to the MHD kink instability as an eruption driver. However, they also pointed out that both the brightenings in the TRACE $1600 \AA$ channel and the bipole emergence were suggestive of initial magnetic reconnection beneath the filament. This initial reconnection then triggered reconnection above the filament as suggested by a quadrupolar topology and the brightening of UV ribbons, a scenario consistent with the internal tethercutting model. Furthermore, the location and timing of the X-ray preflare brightening observed in our analysis (close to the PIL, before the slow-rise of the filament) support the view of internal tether-reconnection as an initial trigger.

\subsection{May 27 (event 5: limb)}

The event on 2002 May 27 at the western solar limb (10N 87W) was associated with a GOES M2.0 class flare (Fig. A.3). This event has been reported as "failed" by Ji et al. (2003) and, more recently, by Alexander et al. (2006). Indeed, the motion of the filament seen in TRACE images was observed to stop $\sim 80 \mathrm{Mm}$ above the photosphere ( $\mathrm{Ji}$ et al. 2003) and there was no detectable CME in the LASCO/C2 data. However, we observed a cavity eruption in Mark-IV (MK4) images taken by the Mauna Loa Solar Observatory (MLSO). 
Both studies report a HXR (12-25 keV) coronal source above the filament (Fig. 13), starting around 18:01 UT, at the beginning of the filament's slow-rise. The slow-rise motion was observed between 18:01-18:02 UT, followed by a fast expansion with a distinctive untwisting motion.

In our analysis of this event, we observed the preflare emission detected with RHESSI in the 12-25 keV energy band, prior to the impulsive phase onset, between 17:52-18:00 UT. RHESSI images of the preflare X-ray brightening between 17:54-17:57 UT revealed the presence of a 12-25 keV source on the opposite (eastern) hemisphere of the Sun (at $x=-850^{\prime \prime}$ and $\left.y=250^{\prime \prime}\right)$. This second source must contribute to the preflare emission detected by GOES. Therefore, in this case, we are not able to establish a correlation between the preflare X-ray brightening seen in the spatially integrated X-ray light-curves and the filament eruption.

We confirm the first HXR source observed above the erupting footpoint of the filament at the beginning of the filament slow-rise (Fig. A.3), as well as a second coronal HXR source which has been identified by Alexander et al. (2006) under the apex of the filament during the fast-rise phase. Alexander et al. (2006) have argued that this second source implies ongoing magnetic reconnection in a current sheet formed via a kink instability. These findings support the MHD simulations by Gibson \& Fan $(2006 \mathrm{a}, \mathrm{b})$ and observables predicted by Tripathi et al. (2007). As with the eruption on 2004 November 10 (event 4 of Table 1), we agree with the possibility that the filament may have been driven by a kink instability during its eruption after the slow-rise onset. However, the first HXR source suggests tethercutting reconnection (external in this case) as the initial release mechanism.

\subsection{April 16 (event 6: limb)}

The filament eruption on 2002 April 16 occurred on the western solar limb $(27 \mathrm{~N} 80 \mathrm{~W})$, in association with a GOES M2.5 flare peaking at 13:18 UT, and a CME detected by LASCO/C2 at approximately 13:50 UT. The event has been previously analysed by Sui et al. (2004) and Goff et al. (2005). The GOES and RHESSI light-curves for this event show a conspicuous preflare enhancement which peaked at approximately 12:58 UT (Fig. A.4). The preflare X-ray brightening also manifested itself as an EUV enhancement in TRACE $195 \AA$ images, when the end of a loop arcade system started to brighten (around 12:55 UT). We imaged the preflare $12-25 \mathrm{keV}$ X-ray source at 12:58 UT and confirm the presence of a nonthermal coronal source previously reported by Goff et al. (2005). The preflare source is observed above the filament, suggesting an external-tether cutting eruption mechanism. Sui et al. (2004) imaged the event only after 13:03 UT, approximately 2 min after the impulsive phase onset, thus omitting the preflare phase.

Sui et al. (2004) noticed a decrease in altitude of the coronal source emission after the flare onset (from 13:06 UT onward). Additionally, we discern a dip of $\sim 7 \mathrm{Mm}$ in the altitude of this coronal source (10-15 keV, Fig. 5 in Goff et al. 2005), earlier in the height-time profile, between approximately 12:58 UT and 13:01 UT. A downward motion of the coronal source, not predicted by current flare models, has been identified in several flares (e.g. Sui et al. 2004; Veronig et al. 2005; Holman et al. $2005)$ previously imaged with RHESSI. This motion was observed during the early flare phases, before then changing to the commonly observed upward expansion of the flare loop system.
We observed the preflare X-ray source above the filament channel located close (within 20") from the main energy emission site indicated by RHESSI 12-25 keV contours taken during the flare impulsive phase at 13:12 UT (Fig. A.4, bottom panel).

\subsection{November 24 (event 7: on-disk)}

The filament eruption on 2000 November $24(21 \mathrm{~N} 15 \mathrm{~W})$ has been studied by Moon et al. (2004), who reported several transient brightenings in $\mathrm{H} \alpha$ and, more noticeably in TRACE $1600 \AA$ images before the X1.8 flare peaking at 21:59 UT. The UV preflare brightenings (Fig. A.5, bottom left panel) occurred near one footpoint of the erupting filament and were also associated with canceling magnetic features (CMFs) observed in the MDI magnetograms (Fig. A.5, top right panel). The UV preflare activity was reported close to the PIL (we estimate within $5^{\prime \prime}$ distance). Moon et al. (2004) suggest that flux variations of these CMFs are an indication of the flux cancelation having been driven by the emergence of the new flux. It was noted that the filament slowrise onset at 21:31 UT coincided with the preflare UV brightenings and CMFs. We found that a small X-ray enhancement which peaked at 21:34 UT in the GOES X-ray light-curves is coincident with this preflare activity. We also note that the $\mathrm{H} \alpha$ brightenings reported by Moon et al. (2004) provide evidence for nonthermal preflare emission. We observed the preflare UV brightenings located within $40^{\prime \prime}$ from the main energy emission site, which is marked in the EIT $195 \AA$ image taken at $\sim 21: 47$ UT in Fig. A.5, bottom right panel. These observations are suggestive of an internal tether-cutting eruption mechanism for which the emerging flux may be a trigger.

\subsection{July 11 (event 8: limb)}

The erupting filament on 1998 July 11 (Fig. A.6) occurred close to the western solar limb $(32 \mathrm{~S} 68 \mathrm{~W})$ and was accompanied by a C1.7 class flare. The filament eruption looks "symmetric" according to the definition of Tripathi et al. (2006a), rising up from the centre, with one initially anchored footpoint in the middle of the filament channel. This event has been analysed in detail by Sterling \& Moore (2005), who reported brightenings in TRACE $195 \AA$ images beginning concurrently with the start of the filament's slow-rise (at 04:22 UT) beneath the rising filament. It was noted, however, that while the slow-rise was obvious from this time, an "agitation" (activation) was present earlier, when their observations started at 04:10 UT. We observe the preflare brightenings under the filament's initially anchored footpoint as indicated in Fig. A.6 (bottom panel).

We observe a preflare X-ray brightening in the GOES 0.5-4 A channel peaking at 04:23 UT. The SXR brightenings were observed by Sterling \& Moore (2005) in Yohkoh/SXT images at the same time and location as the EUV brightenings. Nonthermal HXR emission from Yohkoh/HXT was first detected at 04:32 UT, after the start of the impulsive phase and fast-rise phase of the filament. The preflare as well as the later main flare emission were observed within a small region of approximately $20^{\prime \prime}$ marked in Fig. A.6, bottom panel.

Sterling \& Moore (2005) showed Kitt Peak magnetograms taken from 1998 July 9 with overlayed TRACE and SXT images. This shows the location of the preflare brightenings close to the PIL (within 10"). Newly emerged flux near the location of the initial brightenings was also reported by Sterling \& Moore (2005) about 2 days prior to the event. 
Sterling \& Moore (2005) concluded that their observations were consistent with an internal tether-cutting model, in which the emerging flux was a catalyst of the eruption onset. We note that although there was no available coronographic data for this event, we can say that this filament eruption was most probably associated with a CME, based on the formation of EUV posteruption arcades, as studied by e.g. Tripathi et al. (2004).

\section{Summary of results}

We have studied the spatial and temporal relationships between $\mathrm{X}$-ray preflare brightenings and filament eruptions. The results derived from the analysis of the 8 sampled events are summarised below:

1. In at least seven out of eight events, we found the small preflare X-ray bursts spatially and temporally correlated with the filament eruption. Therefore, we refer to these preflare brightenings as precursors to the flare and filament eruption. In a double event (on 2005 July 30, event 2 of Table 1), the first filament eruption and flare appeared as a precursor to the second, and more impulsive eruption.

2. The precursors were observed between 2 and 50 minutes before the start of the filament fast-rise and flare impulsive phase. They appear to be discrete, spatially localised instances of energy release, rather than a gradual enhancement leading to the main energy release.

3. In five events $(2,3,4,7$ and 8 of Table 1$)$, the X-ray precursors were first observed beneath the filament, close to the initially anchored footpoint. In two events (1 and 6 of Table 1) the precursors appeared as HXR coronal sources situated above the filament. In one event ( 5 of Table 1$)$ we could not establish a correlation between the X-ray precursor and the filament eruption.

4. The filaments begin to rise from the location of the precursor. When the filament begins to rise from one observed (initially anchored) footpoint (all events except for event 6), the precursor is located close to this footpoint. In one event (event 1) the HXR precursor traveled with a velocity of $156 \mathrm{~km} \mathrm{~s}^{-1}$ along a PIL, in the same direction as the filament eruption, but on a neighbouring PIL.

5. Magnetic field observations (for events 1, 2, 4, 7 and 8) revealed that the precursors were located on or near the PIL (within 10"). Coinciding with the initial location of the precursors, we observed emerging (events 1, 4 and 8) and canceling (events 2 and 7) magnetic flux. However, the canceling flux may have been driven by emerging flux.

6. Along with thermal sources that produced SXR and EUV emission, nonthermal HXR precursor emission was detected in five $(1,3,4,5,6$ of Table 1$)$ out of the six events observed with RHESSI.

7. In each of the 8 events, the fast-rise phase of the filament eruption started either at the same time as the onset of the impulsive rise of the main flare or up to $5 \mathrm{~min}$ later.

\section{Discussion and conclusions}

Observed in multiwavelength, high-temporal and spatial resolution data, the precursor activity can provide insights into the possible trigger of the filament eruption and associated flare. The $\mathrm{X}$-ray precursors were observed in the form of weak but discrete events (not merely a gradual pre-enhancement to the main flare), similar to precursors of flare-associated CMEs observed by (e.g. Harrison 1986). Early observations of SXR emission prior to the onset of HXR emission during the impulsive flare phases (Harrison 1986; Zhang et al. 2001) have suggested that such flare precursors may accompany the launch of the associated CME. These appear similar to the X-ray precursor brightenings associated with the filament eruptions discussed in this paper.

Using the linear extrapolation method, it has been found that the onset time of a CME coincided with a flare precursor (a small flare) tens of minutes before the main flare (Harrison et al. 1985; Simnett \& Harrison 1985; Harrison 1986). However, this extrapolation method is uncertain since it assumes that the CME has a constant speed from its launch site at the solar surface through the altitude of the coronograph observations. A more reliable analysis was performed by Zhang et al. (2001), who determined the initial evolution of the CME directly using EIT and LASCO/C1 images. This study also found that the CME was initiated earlier than the onset of the main flare during a "preflare" phase, a small enhancement in the X-ray flux ahead of the flare impulsive phase. A more recent benchmark study for CME initiation proposed by Harrison \& Bewsher (2007) found consistent results with the interpretation of Zhang et al. (2001). It is the preflare X-ray activity which is the focus in our paper. Our analysis of eight events provides evidence of the spatial and temporal correlation between the filament eruption onset and X-ray precursors.

For the first time, we have reported preflare HXR brightenings traveling rapidly along a PIL, in the same direction as the asymmetrically erupting filament, but on a separate nearby PIL (event 1 of Table 1). This motion is observed prior to the main flare which occurred on the same PIL, starting within $20^{\prime \prime}$ of the first preflare X-ray brightenings some $15 \mathrm{~min}$ later. This sequence of events is suggestive of the preflare brightenings being related to the trigger of the main energy release. The same correlation may also be present in the case of a double flare and filament eruption (event 2 of Table 1), when the smaller flare appeared to trigger the subsequent main energy release.

Most detailed analyses of filament eruptions reported in the literature focus mainly on the X-ray emission after the filament eruption onset (e.g. Ji et al. 2003; Alexander et al. 2006), with little attention paid to the X-ray emission prior to the flare onset and filament slow-rise. In this paper, we have revisited six such events (and two new events), pointing out the observable signatures for the eruption trigger. The X-ray flare precursors and the pre-eruptive filament evolution also appear as pre-CME symptoms. By investigating the very early stages of filament eruptions in the form of X-ray brightenings, we recognise the potential of this pre-flare activity as a signature of CME initiation.

The precursor X-ray brightenings suggest that initially localised magnetic reconnection events are a common trigger for both flare emission and filament eruption. Like Fárník et al. (2002), we found that the precursors are located along the PIL and they can display nonthermal emission. Our observations of eruptions beneath the filament (five out of eight events) appear consistent with the scenario involving reconnection between the emerging field and the sheared core magnetic field supporting the filament (internal tether-cutting). However, at present, we cannot exclude an external tether-cutting (or "breakout") scenario, when precursor HXR brightenings were clearly observed above the loop-top high in the corona (events 5 and 6). One filament eruption (event 4) suggested that internal tether-cutting reconnection may be followed closely by external tether-cutting reconnection, with the magnetic tethers being weakened first at low altitudes, and then later at greater altitudes above the filament (Williams et al. 2005). 
In all 8 analysed events, the fast-rise phase of the filament started either at the same time as the onset of the impulsive rise of the main flare in the GOES X-ray flux, or a few minutes later. This result indicates that the fast rise phase of the eruption was not triggered only or mainly by an ideal MHD instability, or by loss of magnetostatic equilibrium, acting in the absence of significant tether-cutting reconnection. This timing result, together with the observed initial heating and nonthermal emission during the X-ray precursors, indicate that tether-cutting reconnection was occurring at or before the onset of the fast rise of the filament. While many erupting filaments/flux-ropes appear to kink as they erupt (e.g. Rust \& LaBonte 2005; Török \& Kliem 2005), we are aware of no conclusive observational evidence that this eruption mechanism acts on its own. Initiation by an MHD instability without any field weakening by reconnection could be proved by observations of strong-field filament eruptions in which the impulsive phase is observed prior to any sign of X-ray or EUV brightening in the active region. We know of no such events (Moore \& Sterling 2006). However, evidence has been found of kinking filament eruptions being released by initial tether-weakening involving reconnection. Events 4 and 5 of Table 1 are two examples of such eruptions. Earlier observations reported by Wang \& Shi (1993) showed a filament eruption characterised by an untwisting upward motion associated with a localised precursor "microflare" representing the first signature for the disturbance of the system. In conformity with the conclusions drawn from event 4 discussed in this paper, Wang \& Shi (1993) found that canceling magnetic reconnection beneath the filament can be responsible for the untwisting and upward motion.

The precursors were found on or near the PIL, but the question remains open as to what creates these brightenings. As predicted by the internal tether-cutting models (Moore \& Roumeliotis 1992), this could be due to reconnection between the newly emerging flux at the PIL and the pre-existing flux. Based on observations (Feynman \& Martin 1995; Wang \& Sheeley 1999; Tripathi 2005) and MHD simulations (Chen \& Shibata 2000; Lin et al. 2001), it has been found earlier that magnetic reconnection between the emerging flux and the preexisting flux can lead to the CME eruption. From our observations we found the new flux emerging at the same location as that of the precursor brightenings. However, the active regions were highly complex, and it was difficult to distinguish between flux emergence and flux cancelation (which may have been driven by the emerging flux). In either case, we believe that, eventually, both flux emergence and flux cancelation have a similar effect, that is they can both result in magnetic reconnection leading to loss of equilibrium.

During the multiwavelength analysis carried out in our work, we became aware of the advantages and disadvantages associated with observations from different instruments. The analysis of events located on-disk was affected by projection effects which prevented us from making conclusive observations about the precursor location and the slow-rise motion of these filaments. However, by choosing filament eruptions located on-disk (e.g. events 1 and 2), we gained the advantage of tracking the geometry and evolution of the magnetic fields. Most early studies of preflares remained inconclusive due to lack of suitable observations. We used TRACE observations of limited FOV, but which track the evolution of the erupting EUV filaments with highcadence and spatial resolution. The temporal evolution of X-rays during the events was obtained from GOES. These satellites have the advantage of continuous temporal coverage; they detect the integrated emission, but lack spatial information about the precursors. Complementing these observations, RHESSI X-ray images and spectra brought insight into the location and spatial extent of the event precursors and their associated emission mechanisms. There is no doubt that the problem of finding the solar eruption trigger can only be solved with the aid of suitable multiwavelength observations to constrain theoretical models. The recently launched solar missions, STEREO and Hinode, and the future Solar Dynamics Observatory (SDO), promise improved observations to provide further insight into the destabilisation trigger of solar eruptions.

Acknowledgements. We thank the anonymous referee for useful comments and suggestions that have improved the quality of the manuscript. The authors are grateful to Alphonse Sterling and Ronald Moore for providing the cartoons adapted in Fig. 1. We thank Karel Schrijver for his help with the analysis of TRACE data. TRACE and RHESSI are NASA Small Explorer projects. Vasyl Yurchyshyn and the BBSO staff are acknowledged for providing BBSO H $\alpha$ images. C.C. is grateful for scholarship support received from the University of Cambridge Overseas Trust, an Isaac Newton Studentship from the Cambridge Institute of Astronomy and an Overseas Research Student Award. D.T. and H.E.M. acknowledge support from STFC.

\section{References}

Alexander, D., Liu, R., \& Gilbert, H. R. 2006, ApJ, 653, 719

Antiochos, S. K. 1998, ApJ, 502, L181

Antiochos, S. K., DeVore, C. R., \& Klimchuk, J. A. 1999, ApJ, 510, 485

Asai, A., Nakajima, H., Shimojo, M., et al. 2006, PASJ, 58, 1, L1

Bumba, V., \& Křiviský, L. 1959, Bull. Astron. Inst. Czech, 10, 221

Chen, P. F., \& Shibata, K. 2000, ApJ, 545, 524

Cheng, C.-C. 1990, ApJ, 349, 362

Cheng, C.-C., Pallavicini, R., Acton, L. W., \& Tandberg-Hanssen, E. 1985, ApJ, 298,887

Chifor, C., Mason, H. E., Tripathi, D., et al. 2006, A\&A, 458, 965

Contarino, L., Romano, P., Yurchyshyn, V. B., \& Zuccarello, F. 2003, Sol. Phys., 216,173

Delaboudinière, J.-P., Artzner, G. E., Brunaud, J., et al. 1995, Sol. Phys., 162, 291

Dere, K. P., Brueckner, G. E., Howard, R. A., et al. 1997a, Sol. Phys., 175, 601

Dere, K. P., Landi, E., Mason, H. E., Monsignori Fossi, B. C., \& Young, P. R. 1997b, A\&AS, 125, 149

Fan, Y. 2005, ApJ, 630, 543

Fárník, F., \& Savy, S. K. 1998, Sol. Phys., 183, 339

Fárník, F., Hudson, H. S., Karlický, M., \& Kosugi, T. 2002, A\&A, 399, 1159

Feynman, J., \& Martin, S. F. 1995, J. Geophys. Res., 100, 3355

Gallagher, P. T., Dennis, B. R., Krucker, S., Schwartz, R. A., \& Tolbert, A. K. 2002, Sol. Phys., 210, 341

Gibson, S. E., \& Fan, Y. 2006a, J. Geophys. Res., 11112103

Gibson, S. E., \& Fan, Y. 2006b, ApJ, 637, L65

Goff, C. P., van Driel-Gesztelyi, L., Harra, L. K., Matthews, S. A., \& Mandrini,

C. H. 2005, A\&A, 434, 761

Grigis, P. C., \& Benz, A. O. 2005, ApJ, 625L, 143

Handy, B. N., Acton, L. W., Kankelborg, C. C., et al. 1999, Sol. Phys., 187, 229 Harrison, R. A. 1986, A\&A, 162, 283

Harrison, R. A., \& Bewsher, D. 2007, A\&A, 461, 1155

Harrison, R. A., Waggett, P. W., Bentley, R. D., et al. 1985, Sol. Phys., 97, 387

Holman, G. D., Sui, L., Schwartz, R. A., \& Emslie, A. G. 2003, ApJ, 595, L97

Holman, G. D., Sui, L., Brosius, D. G., \& Dennis, B. R. 2005, AGU, Fall Meeting, SH13A-0288

Hurford, G. J., Schmahl, E. J., Schwartz, R. A., et al. 2002, Sol. Phys., 210, 61 Isobe, H., \& Tripathi, D. 2006, A\&A, 449, L17

Isobe, H., Tripathi, D., Asai, A., \& Jain, R. 2007, Sol. Phys., submitted

Ji, H., Wang, H., Schmahl, E. J., Moon, Y.-J., \& Jiang, Y. 2003, ApJ, 595, L135 Kai, K., Nakajima, H., \& Kosugi, T. 1983, PASJ, 35, 285

Landi, E., Del Zanna, G., Young, P. R., et al. 2006, ApJS, 162, 261

Lin, R. P., Dennis, R. B., Hurford, G. J., et al. 2002, Sol. Phys., 210, 3

Lin, J., Forbes, T. G., \& Isenberg, P. A. 2001, J. Geophys. Res., 106, 25053

Linker, J. A., Mikić, Z., Riley, P., Lionello, R., \& Odstrcil, D. 2003, AIPC, 679, L703

Martin, S. 1980, Sol. Phys., 68, 217

Moon, Y.-J., Chae, J., Choe, G. S., et al. 2004, JKoAS, 37, 41

Moore, R. L. 1998, ApJ, 324, 1132

Moore, R. L., \& Roumeliotis, G. 1992, in Eruptive Solar Flares, ed. Z. Svestka et al., 399, 69 
Moore, R. L., \& Sterling, A. C. 2006, in Solar Eruptions and Energetic Particles, ed. N. Gopalswamy (Washington DC: AGU)

Moore, R. L., Sterling, A. C., Hudson, H. S., \& Lemen, J. R. 2001, ApJ, 552, 833

Nagashima, K., Isobe, H., Yokoyama, T., et al. 2007, ApJ, accepted

Phillips, K. J. H., Chifor, C., \& Landi, E. 2005, ApJ, 626, 110

Phillips, K. J. H., Chifor, C., \& Dennis, B. R. 2006, ApJ, 647, 1870

Priest, E. R., \& Forbes, T. G. 2002, A\&ARv, 10, 313

Priest, E. R., Hood, A. W., \& Anzer, U. 1989, ApJ, 344, 1010

Rust, D. M., \& LaBonte, B. J. 2005, ApJ, 622, L69

Scherrer, P. H., Bogart, R. S., Bush, R. I., et al. 1995, Sol. Phys., 162, 129

Schmahl, E. J., Webb, D. F., Woodgate, B., et al. 1989, in Energetic Phenomena on the Sun, ed. M. R. Kundu et al. (Dordrecht: Kluwer Academic Publishers), 77

Shibata, K. 1999, Ap\&SS, 264, 129

Simnett, G. M., \& Harrison, R. A. 1985, Sol. Phys., 99, 291

Sterling, A. C., \& Moore, R. L. 2004b, ApJ, 613, 1221

Sterling, A. C., \& Moore, R. L. 2005, ApJ, 630, 1148

Sterling, A. C., Moore, R. L., \& Thompson, B. J. 2001, ApJ, 561, L219

Sterling, A. C., Moore, R. L., \& Harra, L. K. 2006, SPD meeting, 37, 8.23

Svestka, Z. 2001, Space Sci. Rev., 95, 135
Subramanian, P., \& Dere, K. P. 2001, 561, 372

Sui, L., Holman, G. D., \& Dennis, B. R. 2004, ApJ, 612, 546

Sui, L., Holman, G. D., \& Dennis, B. R. 2006, ApJ, 157, L160

Tappin, S. J. 1991, A\&AS, 87, 277

Török, T., \& Kliem, B. 2005, ApJ, 630, L97

Tripathi, D. 2005, Ph.D. Thesis University of Göttimgen, Copernicus GMBH

Tripathi, D., Bothmer, V., \& Cremades, H. 2004, A\&A, 422, 337

Tripathi, D., Isobe, H., \& Mason, H. E. 2006a, A\&A, 453, 1111

Tripathi, D., Del Zanna, G., Mason, H. E., \& Chifor, C. 2006b, A\&A, 460, L53

Tripathi, D., Gibson, S. E., Qiu, J., et al. 2007, A\&A, in preparation

van Ballegoojen, A. A., \& Martens, P. C. H. 1989, ApJ, 343, 971

Van Hoven, G., \& Hurford, G. J. 1984, Adv. Space Rev., 4, 95

Veronig, A. M., Vršnak, B., Karlicý, M., et al. 2005, Hvar Observatory Bulletin, 29(1), 127

Wang, H., Liu, C., Jing, J., \& Yurchyshyn, V. 2007, ApJ, submitted

Wang, J., \& Shi, Z. 1993, ASP Conf. Ser., 46, 397

Wang, Y.-M., \& Sheeley, N. R., Jr. 1999, ApJ, 510, 157

Williams, D. R., Török, T., Démoulin, P., van Driel-Gesztelyi, L., \& Kliem, B. 2005, ApJ, 628, L163

Zhang, J., Dere, K. P., Howard, R. A., Kundu, M. R., \& White, S. M. 2001, ApJ, 559,452 
C. Chifor et al.: X-ray precursors to flares and filament eruptions, Online Material $p 1$

\section{Online Material}


C. Chifor et al.: X-ray precursors to flares and filament eruptions, Online Material $p 2$

\section{Appendix A: Figures describing events 3-8 of Table 1}
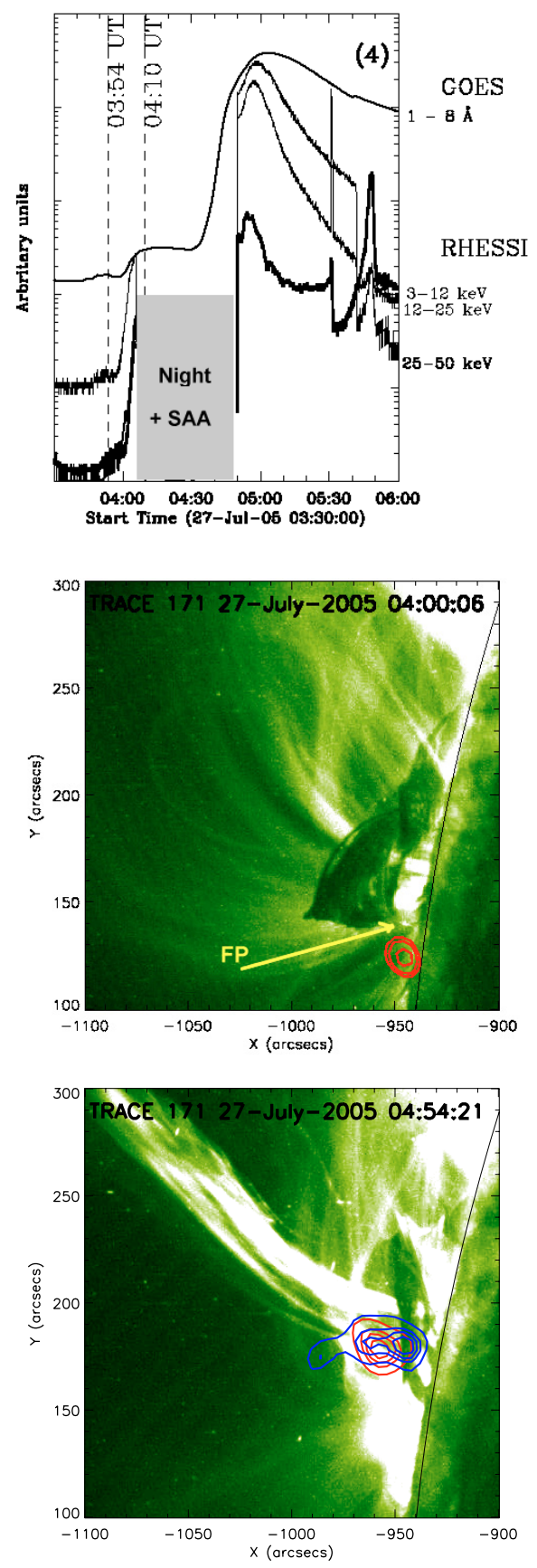

Fig. A.1. The X-ray flare and erupting filament evolution during event 3 of Table 1. Top panel: GOES fluxes in the 1-8 $\AA$ wavelength band with overlayed RHESSI light-curves. The $y$ axis has a logarithmic scale. Between 04:04 and 04:50 UT RHESSI passed through night-time and SAA. The spikes observed in the RHESSI light-curves after 05:30 UT are due to brief removals of the instrument's thin attenuator which was inserted at around 04:50 UT. We observed two preflare emission peaks at 03:55 UT and 04:10 UT. Middle panel: the erupting filament observed in the TRACE $171 \AA$ filter. The location of the X-ray preflare brightening is marked by RHESSI image contours (6-12 keV emission plotted in red). The X-ray image was taken at 04:01 UT, at the start of the second preflare brightening marked in the GOES plot. The filament footpoint (FP) indicated on the figure (by the arrow) is first observed to slow-rise $\sim 03: 58$, in near synchrony with the first preflare X-ray brightening. Bottom panel: TRACE $171 \AA$ A image of taken at 04:54 UT, during the flare impulsive phase. RHESSI 6-12 keV (red) and $25-50 \mathrm{keV}$ (blue) image contours mark the location of the main energy release. 
C. Chifor et al.: X-ray precursors to flares and filament eruptions, Online Material $p 3$
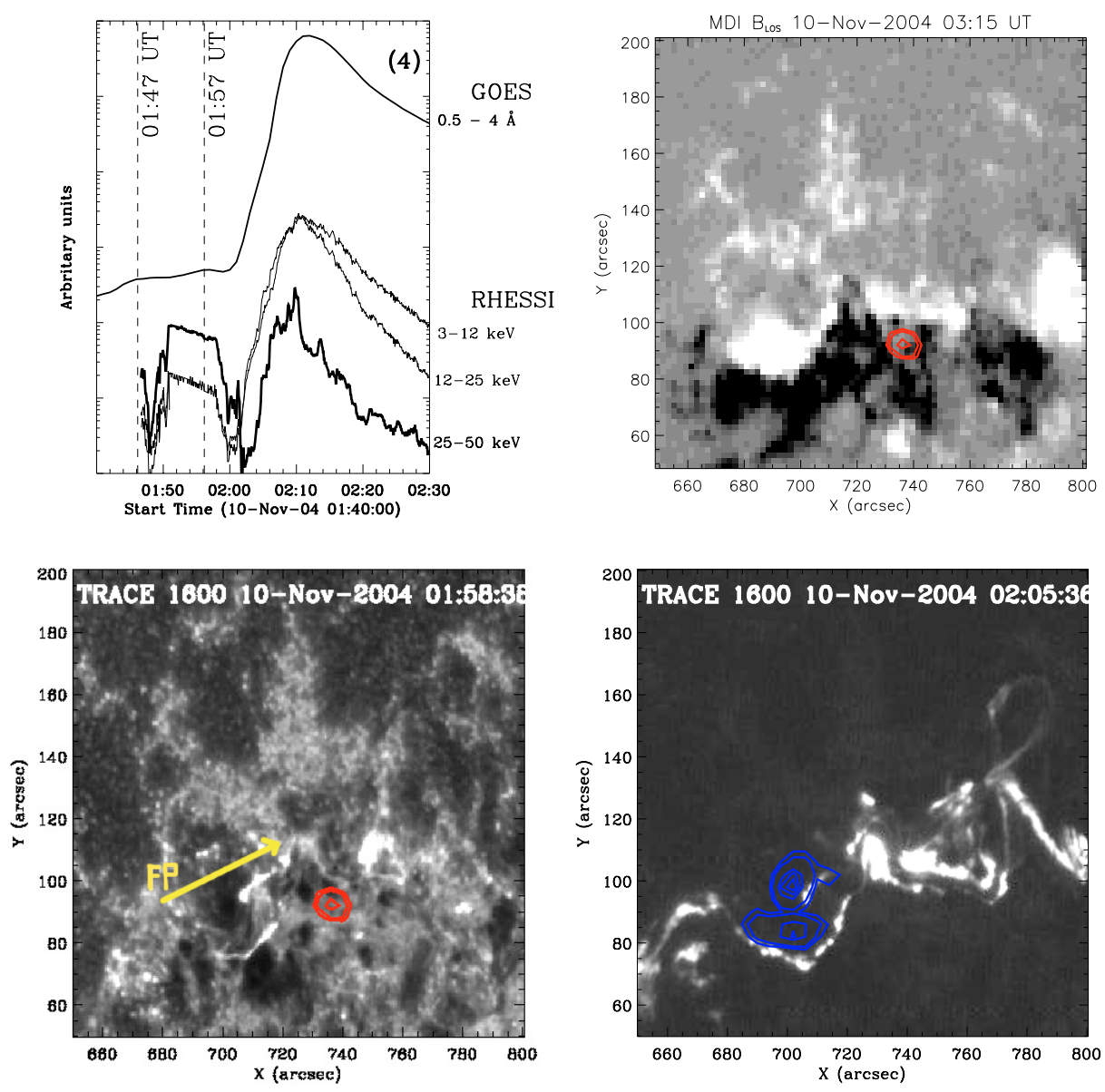

Fig. A.2. The X-ray flare, photospheric magnetic field and the erupting filament during event 4 of Table 1. Top, left panel: GOES light-curves in the 0.5-4 A wavelength band with overplotted RHESSI light-curves in the energy ranges of 3-12 keV, $12-25 \mathrm{keV}$, and $25-50 \mathrm{keV}$ averaged over RHESSI detectors 1, 3, 4, 5, 6, 8 and 9. RHESSI emerges from the SAA around 01:48 UT and observes the preflare and main flare peak emissions with both the thin and thick attenuators in place ("A3" state). The $y$ axis has a logarithmic scale. The preflare X-ray peak emission times (01:47 UT and 01:57 UT) are indicated. Top, right panel: RHESSI 6-12 keV (red) contour image taken around the time of the second preflare peak ( 01:58 UT), overlayed on an MDI image to reveal its location near the PIL (within 10"). Bottom, left panel: image taken prior to the filament eruption at 01:58 UT in the TRACE $1600 \AA$ filter. Overlayed are the same RHESSI 6-12 keV preflare contours. The approximate location of the rising footpoint (FP) of the filament is also indicated. Bottom, right panel: TRACE $1600 \AA$ A image taken during the fast-rise phase of the filament ( 02:05 UT). RHESSI HXR 25-50 keV (blue) contours obtained around the flare peak (02:10 UT) are overplotted to show the site of the main energy release relative to the filament eruption. 
C. Chifor et al.: X-ray precursors to flares and filament eruptions, Online Material $p 4$


Fig. A.3. The X-ray flare and erupting filament evolution during event 5 of Table 1. Top panel: GOES fluxes in the $0.5-4 \AA$ wavelength band with overlaid RHESSI light-curves in the energy ranges of 3-12 keV, 12-25 keV, averaged over RHESSI detectors 1, 3, 4, 5, 6, 8 and 9. Until 18:03 UT, when the thin RHESSI attenuator is inserted, the instrument observes with no attenuators in place. The preflare peak emission time (17:56 UT) is indicated. Middle panel: the filament was observed in the TRACE $195 \AA$ filter prior to eruption at 18:01 UT. Overlaid are simultaneous RHESSI 12-25 keV contours of the HXR coronal source identified at this time. The approximate location of the rising footpoint (FP) of the filament is indicated by the arrow. Bottom panel: TRACE $195 \AA$ image of the erupting filament, during the fast-rise phase. Overlaid RHESSI 12-25 keV contours obtained at approximately the same time (18:07 UT) show the site of the main energy release. The contours include the coronal source previously identified by Alexander et al. (2006) at the crossing location of the filament legs. 
C. Chifor et al.: X-ray precursors to flares and filament eruptions, Online Material $p 5$
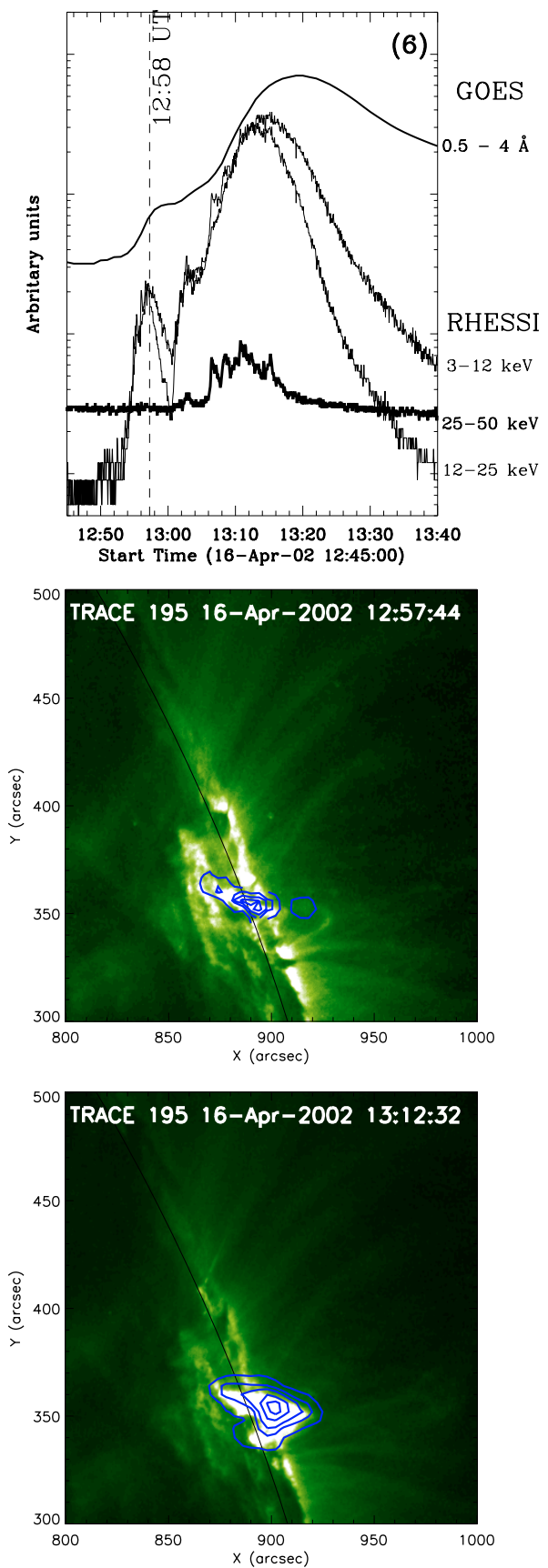

Fig. A.4. The X-ray flare and erupting filament evolution during event 6 of Table 1. Top panel: GOES fluxes in the $0.5-4 \AA$ wavelength band with overlaid RHESSI light-curves in the energy ranges of $3-12 \mathrm{keV}, 12-25 \mathrm{keV}$, and $25-50 \mathrm{keV}$ averaged over RHESSI detectors $1,3,4,5,6,8$ and 9 . No RHESSI attenuators were in place during this event. The $y$ axis has a logarithmic scale. The preflare peak emission time (12:58 UT) is indicated. Middle panel: the filament observed in the TRACE $195 \AA$ filter close to the time of the preflare brightening. RHESSI image contours were taken at the time of the preflare X-ray brightening (12:58 UT) in the 12-25 keV energy band (blue contours). Bottom panel: TRACE $195 \AA$ image of the erupting filament during the fast-rise phase. Overlaid RHESSI 12-25 keV contours obtained at approximately the same time (13:12 UT) show the site of the main energy release. 
C. Chifor et al.: X-ray precursors to flares and filament eruptions, Online Material $p 6$


Fig. A.5. The X-ray flare, erupting filament and photospheric magnetic field during event 7 of Table 1. Top, left panel: GOES fluxes in the 0.5-4 $\AA$ wavelength band. The preflare peak emission time (21:34 UT) is indicated. Bottom left, panel: the erupting filament observed in the TRACE 1600 filter at the time of the X-ray preflare brightening peak. The UV preflare brightenings are encircled and the filament footpoint (FP) is indicated by the arrow (no RHESSI X-ray data available for this event). Top right, panel: the site of the UV preflare brightening is also marked on an MDI magnetogram showing the photospheric magnetic field prior to eruption. Bottom right, panel: EIT $195 \AA$ A image taken during the main phase of the flare at 21:47:29 UT, with the main flare emission site encircled. 
C. Chifor et al.: X-ray precursors to flares and filament eruptions, Online Material $p 7$


Fig. A.6. The X-ray flare and erupting filament evolution during event 8 of Table 1. Top panel: GOES fluxes in the 0.5-4 $\AA$ wavelength band. The preflare emission peak time at 04:23 UT is indicated. Middle panel: the erupting filament observed in the TRACE $195 \AA$ filter during the slow-rise, with the EUV preflare brightening encircled (no RHESSI data available in this case). Bottom panel: the erupting filament observed in a TRACE $195 \AA$ image taken during the fast-rise of the filament and flare impulsive phase. The initially anchored footpoint (FP) located in the middle of the rising filament is indicated. Also indicated is the approximate location of the main flare emission site. For comparison, the same region marked in the middle panel is encircled. 